\title{
Research Article \\ Experimental Results for the Settling Behaviour of Particle-Fiber Mixtures
}

\author{
Markus Feist, Hermann Nirschl, Jörg Wagner, Georg Hirsch, and Samuel Schabel
}

Received 1 October 2007; Accepted 6 December 2007

Recommended by Eiji Iritani

\begin{abstract}
Sedimentation of organic fibres and inorganic particles can be observed in several industrial applications. Fibres are involved not only in wastewater treatment but also in other separation applications. In the paper industry, the separation of inorganic filler and coating particles from short cellulose fibres is still a challenge in the recycling process. During that process, particles have to be removed to obtain a purified fibre suspension. These fibres can be used again to produce new paper. With the currently applied techniques, like screening and flotation, the efficiency of short fibre separation is very poor. Moreover, also separation by sedimentation fails due to similar settling velocities of heavy-small particles and the light and larger fibres. This paper concentrates on the sedimentation of organic fibres and inorganic particles in water. The investigated suspensions are made by resolving two different and specially produced papers a coated and an uncoated one, as well as the single components used for its production. We observe a different sedimentation behaviour according to the concentration of fibres and particles in the suspension and the $\mathrm{pH}$ value of it. The main result is that, according to the fibre rate, the sedimentation behaviour is particle dominant or fibre dominant.
\end{abstract}

Copyright (C) 2007 Markus Feist et al. This is an open access article distributed under the Creative Commons Attribution License, which permits unrestricted use, distribution, and reproduction in any medium, provided the original work is properly cited.

\section{INTRODUCTION}

Knowledge about the sedimentation behaviour of fibres and particles is necessary for many applications. For wastewater treatment, sedimentation is used to clean the fluid phase, the water, from solid phase which consists of particles and fibres. In some filtration processes, fibres are used as filter excipient. Finally, flotation processes are employed in paper recycling, where a separation of inorganic and organic fine material is rather frequently required. These three examples show that it is worth discussing the effects and differences between the sedimentation of fibr suspensions, particle suspensions, and fibre-particle suspensions.

One of the first investigations of a falling sphere was made by Stokes [1]. He developed an analytical formula for a slow falling sphere in a fluid. Several decades later Durlofsky et al. [2] computed the hydrodynamic interactions among numerous suspended particles under the assumption of small Reynolds numbers. Experiments concerning the sedimentation behaviour were made by Beiser [3], Beiser et al. [4], and Bickert [5]. They investigated the sedimentation velocity and particle distribution with the help of a long-arm centrifuge. One result was a sedimentation velocity distribution which not only depends on the concentration of the particles, but also on the $\mathrm{pH}$-value and the ionic strength. In a dilute suspension, they started in the Stokes regime. With further increase of the particle concentration, clusters are formed and the velocity of the agglomerates rises. Further increase leads to a so-called swarm sedimentation, where the particles tend to demix and the whole swarm has the highest velocity. Finally, when the concentration becomes too high, interference and contact effects between single particles slow down the sedimentation of the whole collective. The result is an average velocity much smaller than the Stokes velocity. This regime is used for separation purposes while swarm sedimentation gives the preferred process conditions for classification. The experiments have been performed under the assumption that particles do not agglomerate during the sedimentation process.

A different behaviour was investigated for the sedimentation of fibres. One approach was carried out by Happle and Brenner [6]. They provide an analytical solution for the sedimentation of an elliptical shaped body. A different analytical formula for the sedimentation velocity of fibres was a result of the investigations of Batchelor [7]. His slender body theory postulates that the velocity of a thin but long body can be solved analytically by developing and comparing an inner and an outer mathematical solution. His mathematical solution shows that the direction of the falling body plays a decisive role on the sedimentation velocity.

That is why the core difference between fibre sedimentation compared to particle sedimentation depends on the 
orientation. Kumar and Ramarao [8] investigated the sedimentation of fibres in a dilute regime. They put a fibre suspension in a cylinder and observed the velocity of the separation front between the suspension and the clear fluid. The result is that the higher the concentration, the slower the velocity of the fibre. To avoid boundary effects of the measuring cylinder, its diameter has to be more than one hundred times the length of a fibre. Additionally, it can be seen that fibres tend to agglomerate and align during the sedimentation. These phenomena were also investigated numerically by Butler and Shaqfeh [9] and experimentally by Herzhaft and Guazzelli [10]. They tracked single fibres and found that these not only align and agglomerate but also that the agglomerates have a limited growth, and single fibres oscillate around the vertical direction. All these described investigations were performed with pure particle or pure fibre suspensions. Harlen and Sundararajakumar [11] made experiments with a big sphere in a fibre suspension and establish that the drag coefficient of the sphere has a dependence on the concentration of the fibres.

Overall, the existence of fibres in a suspension has a large influence on the sedimentation of particles. Serious differences are expected for the sedimentation effects of fibreparticle suspensions in comparison to particle-particle or fibre-fibre suspensions. The differences are even higher when the fibres dimensions become similar to the dimensions of the particles. Paper in particular consists of inorganic spheres and organic fine fibres up to $150 \mu \mathrm{m}$. The sedimentation behaviour of such suspensions will be described in this paper.

\section{EXPERIMENTAL TECHNIQUES}

\subsection{Materials}

The analysed paper suspensions were made of a specially produced and finally resolved uncoated and coated papers. The uncoated one consists of organic fibres and $\mathrm{CaCO}_{3}$ as filling material, but has no coating. This paper is the basis of the coated paper which has two additional coatings made with a coating colour which is a $\mathrm{CaCO}_{3}$-kaolin slurry described in Table 1 and $100 \% \mathrm{CaCO}_{3}$ as precoating. The main ingredients are $\mathrm{CaCO}_{3}$ and kaolin. Additionally, several stabilisers are admixed to improve the properties of the paper. A binder is used to fix the particles in the coating colour and onto the fibres. Carboxymethylcellulose and the polyvinyl alcohol regulate the rheological properties of the coating colour and are carriers for the Blancophor which made the paper whiter. Finally, the analysed paper suspensions were made by reslurrying the two different papers in water followed by a separation by a screen with a hole size of $150 \mu \mathrm{m}$. It can be concluded that nearly all particles and fibres in the suspension are smaller than $150 \mu \mathrm{m}$. For simplification, the coated and uncoated papers in the figures and tables refer to the suspensions which are described above. They have the following consistencies (see Table 2). These were chosen to detect differences in the sedimentation velocity distribution, due to coating effects and the fibre-particle proportion of the suspension. The suspension of the coated paper has a low total, only $25 \%$, of organic fibres and a high total, $75 \%$, of inor-
TABLE 1: Contents of the coating colour.

\begin{tabular}{lc}
\hline Material & Percentage/\% \\
\hline $\mathrm{CaCO}_{3}$ & 43.3 \\
Kaolin & 43.2 \\
Binder & 11 \\
Carboxymethyl cellulose & 0.5 \\
Polyvinyl-alcohol & 0.4 \\
Blancophor & 0.6 \\
\hline
\end{tabular}

TABLE 2: Contents of the coated and the uncoated papers' suspension measured by TGA.

\begin{tabular}{lcc}
\hline & Coated paper & Uncoated paper \\
\hline Organic/\% & 25 & 45 \\
Inorganic/\% & 75 & 55 \\
Percentage $\mathrm{CaCO}_{3} / \%$ & 53 & 43 \\
Percentage kaolin $/ \%$ & 22 & 12 \\
\hline
\end{tabular}

ganic particles whereas the suspension of the uncoated paper has a higher total nearly $45 \%$ of fibres and a total of $55 \%$ inorganic particles. Beside the resuspended paper, the single components, that is, the $\mathrm{CaCO}_{3}$, the kaolin slurry, the coating colour, and the filling material, which consists of $\mathrm{CaCO}_{3}$, were investigated to observe differences in the sedimentation behaviour. All the studies were done for mass concentrations varying between $0.5 \mathrm{wt} \%$ to $3.0 \mathrm{wt} \%$ (weight percent). For the particle suspensions, the variation goes up to $20 \mathrm{wt} \%$. Due to the investigation of different agglomerate effects, caused by electrochemical action, the single components and the paper suspensions were analysed at different $\mathrm{pH}$-values and a concentration of $2 \mathrm{wt} \%$. The $\zeta$-potential was measured as well as the sedimentation velocity distribution of the coated paper suspensions.

\subsection{Experimental setup}

One application we used for measurements was the sedimentation balance (Mettler HE10). Due to the small size of the particles, which causes a low-sedimentation velocity, the gravitational force for some of the suspensions has to be increased. Therefore, we used the long-arm centrifuge to get a higher $\mathrm{C}$-value. This measurement technique exploits the pressure difference caused by the particles during sedimentation. A detailed description can be found in Bickert [5] and Stolarski et al. [12].The suspension is put in a cuvette which is at the end of a long centrifuge arm. The pressure difference is measured between two holes in the cuvette. This difference depends on the concentration of particles between the two points, which decreases with increasing time due to particle sedimentation. The final result is a pressure-over-time curve as shown in Figure 1. Additionally, there is the following dependence between the sedimentation velocity distribution and the particle concentration according to the derivative of the pressure-time curve,

$$
Q_{3}\left(u_{p}\right)=\frac{p\left(t_{1}\right)}{p_{0}}+\frac{t_{1}}{p_{0}}\left|\frac{d p(t)}{d t}\right|_{t_{1}},
$$


where $Q_{3}$ is the cumulative distribution, $u_{p}$ the particle velocity, $p$ the pressure difference, $p_{0}$ the pressure difference of the pure liquid, $t$ the time, and $t_{1}$ a certain point in time. Finally, one obtains a sedimentation velocity distribution. Not only the weight concentration of the whole suspension but also the fibre-particle proportion has been varied to detect the influence of the fibres in the suspension. For comparison of sedimentation velocity distributions measured at different centrifugal forces, they have to be normalised by division by the according $\mathrm{C}$-number, which is defined as

$$
C:=\frac{\omega^{2} r}{g}
$$

where $\omega$ is the angular velocity, $r$ the length of the position vector, and $g$ the gravity.

The setting of different $\mathrm{pH}$-value has been used to change the $\zeta$-potential for investigation of possible agglomeration effects. The variations in the alkaline range were done with $\mathrm{NaOH}$, one molar, and in the acid range with $\mathrm{HNO}_{3}$, also one molar. The $\zeta$-potential was measured by AcoustoSizer II and Pen Kem 501.

\section{RESULTS AND DISCUSSION}

\subsection{Single components}

In Figure 2(a), it is shown that the sedimentation velocity distribution depends on the concentration of the $\mathrm{CaCO}_{3}$ suspension, which is one component of coating colour and has an $\mathrm{x}_{50}$ value of $1.0 \mu \mathrm{m}$. It can be seen that the $\mathrm{CaCO}_{3}$ has nearly the same sedimentation velocity distribution for $3 \mathrm{wt} \%$ and $5 \mathrm{wt} \%$. The average velocity of the suspension with a concentration of $10 \mathrm{wt} \%$ and $20 \mathrm{wt} \%$ is then again slightly faster than of $5 \mathrm{wt} \%$. This is caused by the effect of cluster and swarm sedimentation as also described in Beiser [3]. The measurements were done with a C-value of 200 and a duration of 1000 seconds. Within that time, nearly $80 \%$ of the material were sedimented. In contrast, with the same measurement settings as for $\mathrm{CaCO}_{3}$, only $60 \%$ of the kaolin were sedimented as it is shown in Figure 2(b). This is because the kaolin particles are smaller than the $\mathrm{CaCO}_{3}$ particles. The sedimentation velocity distribution also illustrates that $20 \%$ of the kaolin sediments with almost the same sedimentation velocity, whereas the remaining $80 \%$ has a rate greater than ten times slower. This is caused by the agglomeration properties of the kaolin. Some big agglomerates sediment first while the smaller primary particles sediment relatively slowly. The kaolin is the second component of the coating colour. In Figure 2(b), we compare also the sedimentation behaviour of the components, $\mathrm{CaCO}_{3}$ and kaolin, of the coating colour with the coating colour and the mixture of the pigment components at $3 \mathrm{wt} \%$. In all four slurries, the fastest $20 \%$ has almost the same velocity. Therefore, the size of the bigger agglomerates in the $\mathrm{CaCO}_{3}$ slurry, koalin slurry, coating colour and mixture is the same. But the velocity of $80 \%$, the finer parts, differs from each other. For $\mathrm{CaCO}_{3}$, the average sedimentation velocity is the highest and for kaolin the slowest, as expected. Obviously, the mixture and the coating colour velocity distribution can be found in the middle of both distributions. But it is astonishing that the additional components in the coating colour (see Table 1) have no influence on the sedimentation velocity.

The average sedimentation velocity of the suspension of the uncoated paper, shown in Figure 3 measured by the sedimentation balance, rises with lower concentrations. The reason is the interference of the fibres at higher concentrations. The velocity distribution is monomodal because of the high rate, $45 \mathrm{fi} \%$ (fibre percent), of fibres in the suspension. This amount of fibres causes interference effects which slow down fast particles and agglomerates to the same velocity as the surrounding particles and fibres. The sedimentation velocity distribution of the coated paper suspension, see Figure 4, shows a completely different behaviour compared to the uncoated one. This is because of the higher amount of inorganic particles. The distribution for $0.5 \mathrm{wt} \%, 1 \mathrm{wt} \%$, and $2 \mathrm{wt} \%$ is bimodal, which is shown by the sedimentation balance. The faster parts are the agglomerates of fibres and particles. The slower parts are the high amount of nonagglomerated inorganic particles which have a slower sedimentation velocity. At a concentration of $3 \mathrm{wt} \%$, the velocity distribution shows a monomodal rating. The amount of particles and fibres at $3 \mathrm{wt} \%$ is so high that the interference effects cause the same velocity for all particles. The faster particles accelerate the slower ones whereas the slower particles decelerate the faster ones. Therefore, we get a slower velocity for big particles and a higher velocity for the small ones. The difference between the uncoated and the coated papers can be explained by the rate of fibres in the suspension. The more fibres are in the suspension, the higher is the amount of contacts and interference. During the experiments with the uncoated paper in the sedimentation scales, one observes the settling front of fibres. The suspension was divided into two turbid parts. In the lower part, there were fibres and bigger particles which sedimented relatively fast. The upper part was caused by small particles which are not able to sediment in the gravitational field.

The pressure curves shown in Figure 5 of suspensions which have a fibre rate of $45 \mathrm{fi} \%$ are convex. It is interesting to establish the turning point where the fibre amount is so high that the concave pressure signal, which indicates a particle dominated sedimentation, turns to convex signal indicating a fibre dominated sedimentation. To find out this point, we mixed the uncoated and coated papers to adjust the fibre rate. The convexity of the pressure curves rises with suspensions increasing the fibre rate. Between 40 and $45 \mathrm{fi} \%$, the turning point is reached. It is assumed that due to contact forces the fibres align in vertical direction and agglomerate as also stated in Herzhaft and Guazzelli [10] or Kumar and Ramarao [8]. Therefore, the rate of pressure decrease rises from slow at first to relatively fast at the end of the experiment. Below that turning point, in the particle dominated sedimentation area, we investigate how the sedimentation velocity distribution varied with the rate. These velocity distributions are shown in the Figure 6 depending on the fibre rates between 5 and $40 \mathrm{fi} \%$. The starting suspension is the coated paper with a fibre rate of $24 \mathrm{fi} \%$. As this rate increases, 

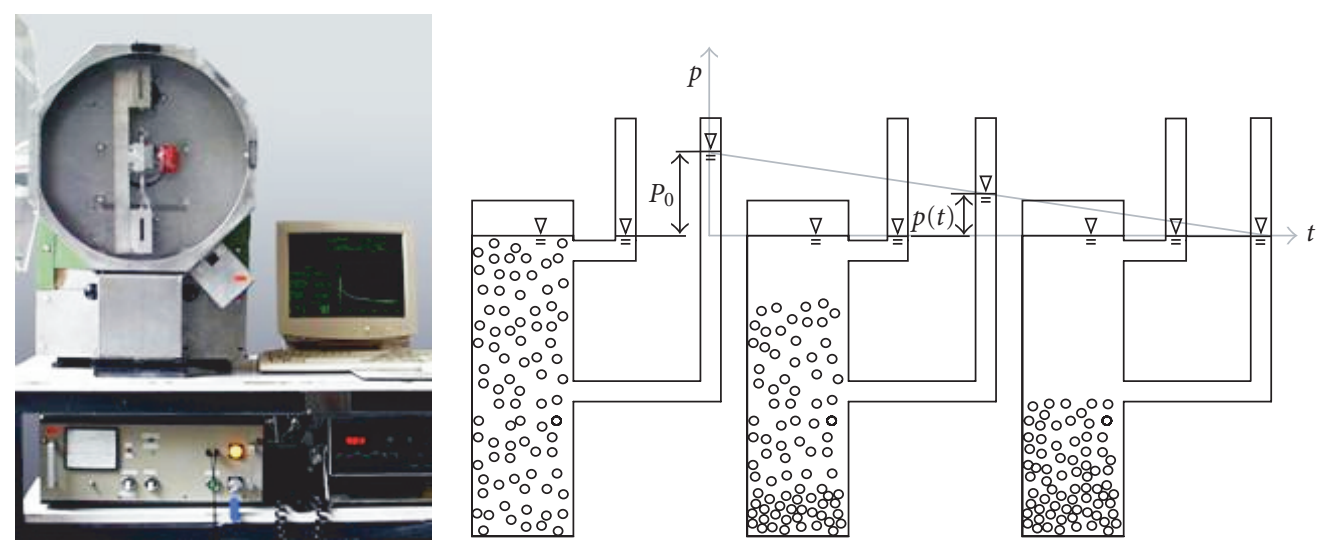

FIGURE 1: Measurement principal of the manometer centrifuge.

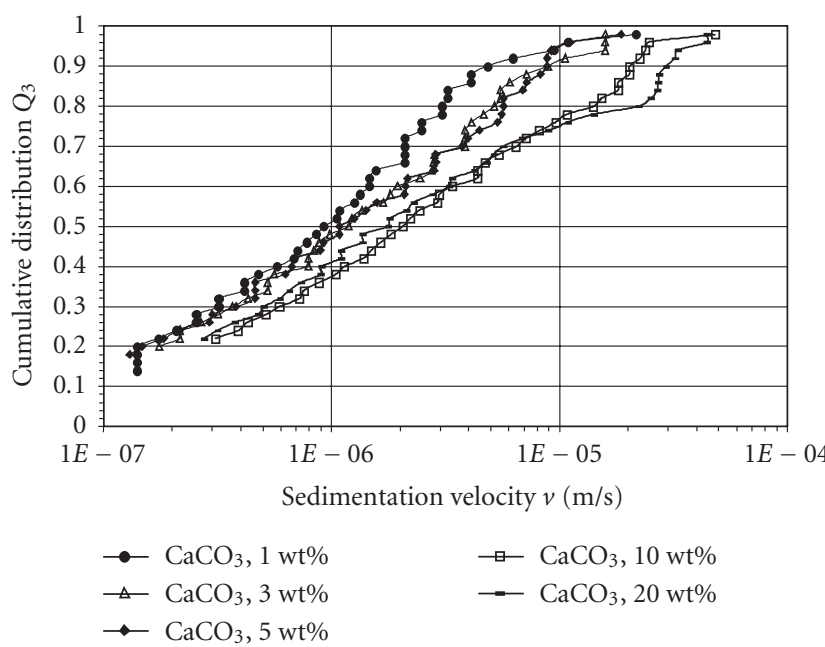

(a)

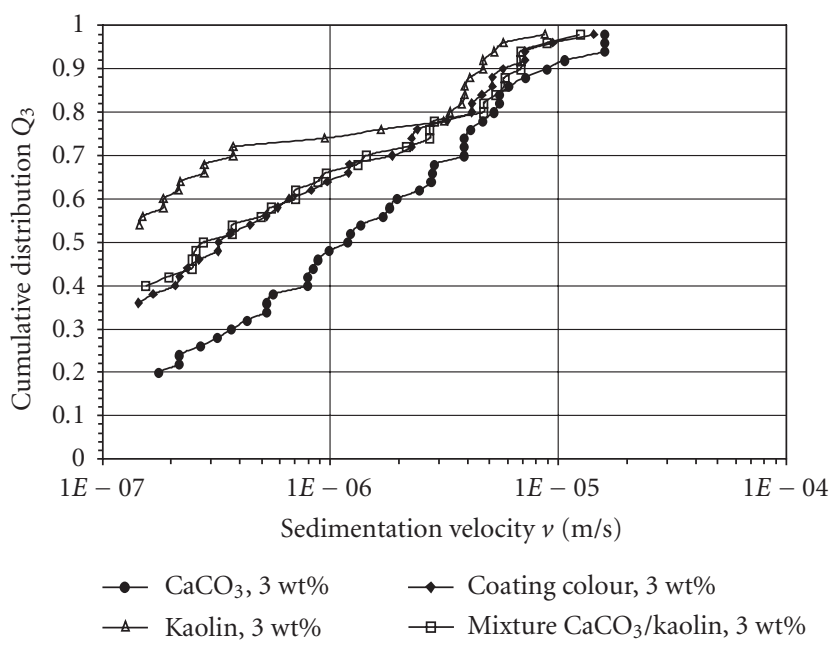

(b)

FIGURE 2: (a) Sedimentation velocity distribution of the $\mathrm{CaCO}_{3}$-suspension at different wt\%. (b) Sedimentation velocity distribution of $\mathrm{CaCO}_{3}$, kaolin, the coating colour, and $\mathrm{CaCO}_{3}$-kaolin mixture at $3 \mathrm{wt} \%$, measured with the long-arm centrifuge. All results are normalised to $g$.

more and more of the solid matter sediment with the same velocity. The average sedimentation velocity becomes higher than for $24 \mathrm{fi} \%$ and decreases from $30 \mathrm{fi} \%$ to $40 \mathrm{fi} \%$. The velocity of the bigger particles decreases while the velocity of the smaller ones increases. At 35 fi\% or 40 fi $\%$ nearly $85 \%$ of the solid matter have the same sedimentation velocity, at $30 \mathrm{fi} \%$, it is only $50 \%$ and at $24 \mathrm{fi} \%$ no area with almost the same sedimentation velocity can be examined. This is due to the increasing interference effects caused by the increasing amount of fibres in the suspension. Between 5 and $24 \mathrm{fi} \%$, the average sedimentation velocity becomes slower, because the less fibres are in the suspension, the less agglomerates will be formed.

In Figure 7, the $\zeta$-potentials of the fibre suspensions and the single components are given. In the acid range, the $\zeta$ potentials of the paper suspensions are positive, the $\mathrm{CaCO}_{3}$ dissolves and the fibres agglomerate. At the original $\mathrm{pH}$-value $(\mathrm{pH} 7.5)$ of the suspension, the $\zeta$-potential of the uncoated paper is higher than that of the single components. In con- trast, the coated paper has a $\zeta$-potential which is between that of the single components. While increasing the $\mathrm{pH}$-value, the $\zeta$-potentials of the single components decrease faster than that of the paper suspensions. At a $\mathrm{pH}$-value around 10, the fillers and the coating colour have a $\zeta$-potential which is lower than that one of the coated paper, which is in turn lower than the $\zeta$-potential of the single fibre component. Because of the higher $\zeta$-potential difference at a $\mathrm{pH}$-value of 10, the agglomerates should be smallerat a $\mathrm{pH}$-value of 7.5. The sedimentation velocity therefore varies extremely when varying the $\mathrm{pH}$-value, see Figure 8 . From the original $\mathrm{pH}$ value at 7.5 to 10 one can observe a velocity nearly 10 times slower. This is due to the reduced agglomeration during the sedimentation process. At a $\mathrm{pH}$-value of 7.0, the velocity is faster, which indicates the formation of larger agglomerates. This corresponds to the observation given in Figure 7. Unfortunately, it was not possible to measure the velocity of the coated paper suspensions in the long-arm centrifuge in the acid range. Because of the fast agglomeration of the fibres, 


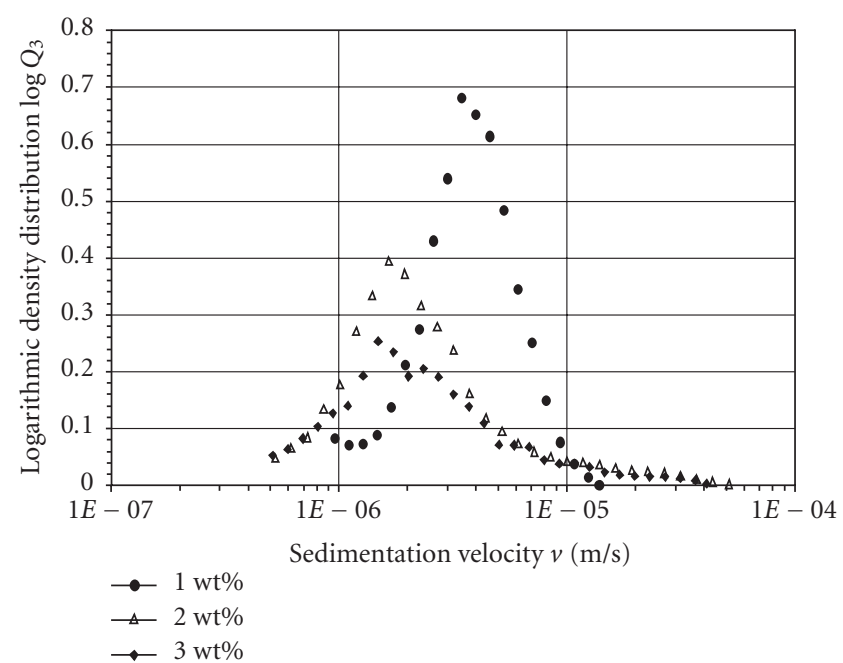

FIgURE 3: Sedimentation velocity distribution of the uncoated paper, at different $\mathrm{wt} \%$, measured with the sedimentation scales.

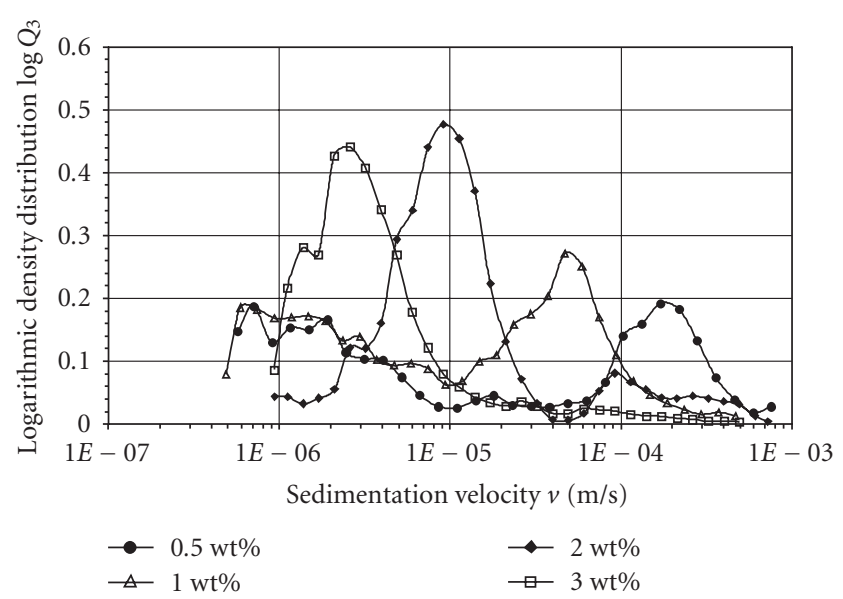

FIGURE 4: Sedimentation velocity distribution of the coated paper, at different wt $\%$, measured with the sedimentation scales.

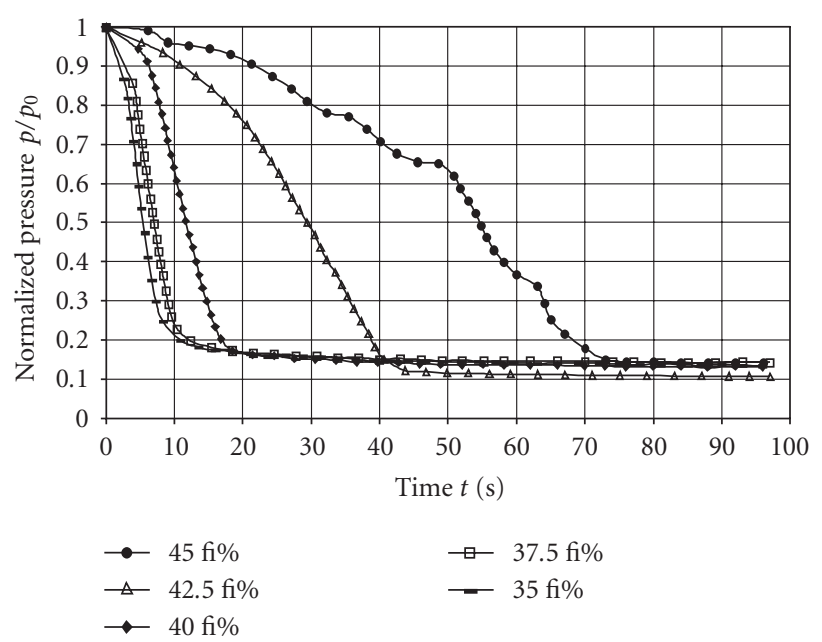

FIgURe 5: Pressure-time curve of a paper mixture of coated and uncoated papers, $2 \mathrm{wt} \%$, measured with the long-arm centrifuge. The results are normalised to $g$.

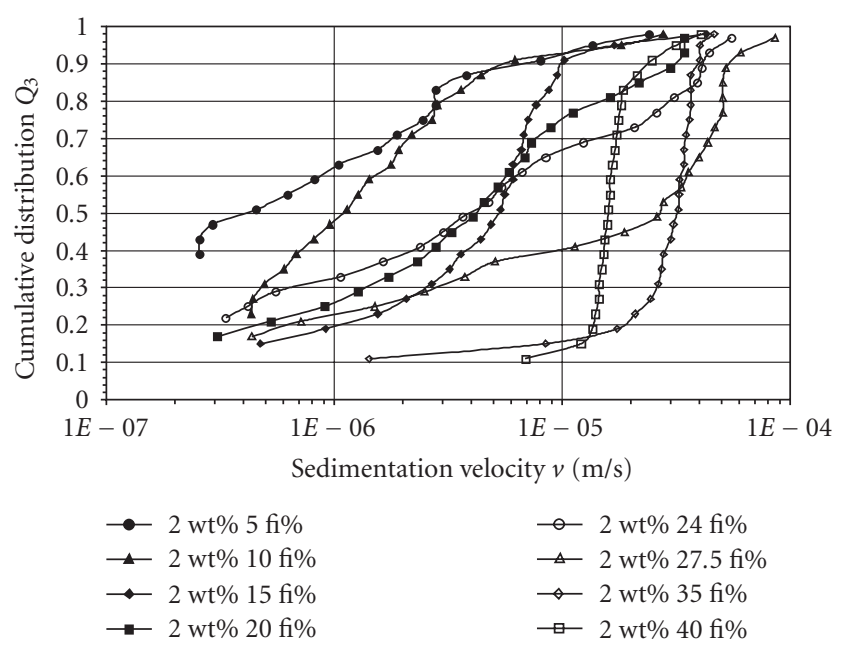

FIGURE 6: Sedimentation velocity distribution of a mixture of uncoated and coated papers, at $2 \mathrm{wt} \%$ and at a different fibre rate, measured with the long-arm centrifuge. The results are normalised to $g$.

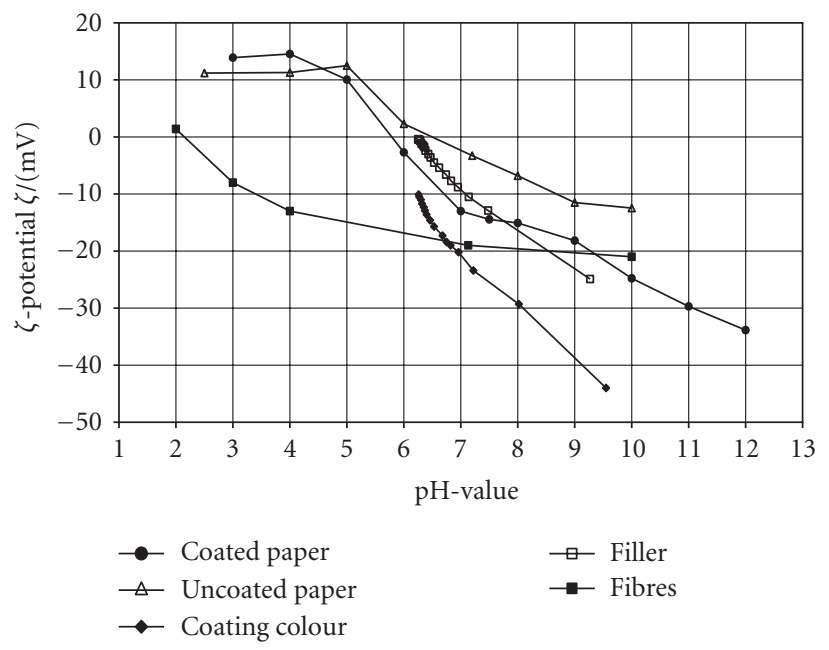

FIGURE 7: $\zeta$-potential of the coated and uncoated papers and their $\mathrm{x}_{50}$-values at different $\mathrm{pH}$-values.

the height of the sediment plugged the lower measurement point.

\section{SUMMARY}

In this paper, we have shown that the sedimentation velocity of fibre-particle suspensions depends on the weight concentration of the solid material, on the fibre-particle ratio and on the $\mathrm{pH}$-value of the suspension. While increasing the weight concentration of the uncoated paper, the interference between the fibres and particles becomes so high that the settling velocity decreases. Furthermore, much solid matter sediments with the same velocity. The coated paper exhibits a different behaviour. Due to the coating particles agglomerating with the fibres, the fibres become heavier and the sedimentation velocity increases. During sedimentation at 


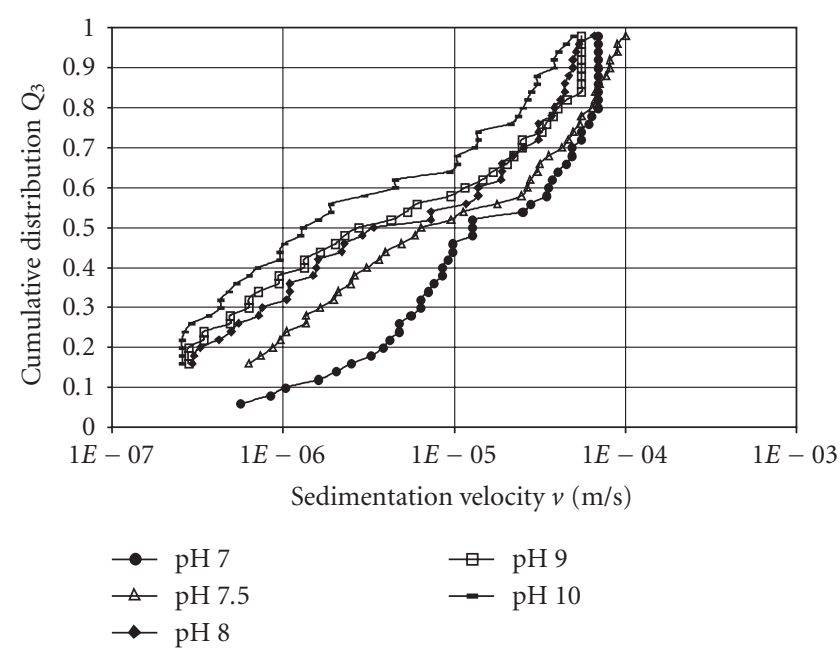

FIGURE 8: Sedimentation velocity distribution of the coated paper at different $\mathrm{pH}$-values.

low-weight concentration, the heavy fibres and big agglomerates sediment first, which causes a bimodal sedimentation velocity distribution. By varying the fibre rate, we discovered that for a suspension mixture of uncoated and coated papers and a fibre rate greater than $40 \%$, the sedimentation is dominated by the fibre effects, such as interference and orientation. Finally, the variation of the $\zeta$-potential by adjusting the $\mathrm{pH}$-value leads to a slower sedimentation velocity for the coated paper suspension because the fibres and particles form less agglomerates.

\section{ACKNOWLEDGMENT}

The authors would like to thank the "Bundesministerium für Wirtschaft und Technologie (BMWi)" and "Arbeitsgemeinschaft Industrieller Forschungsvereinigungen (AIF)" for their financial support.

\section{REFERENCES}

[1] G. G. Stokes, "On the theories of the internal friction of fluids in motion of pendulums," Transactins of the Cambridge Philosophical Society, vol. 9, pp. 8-106, 1851.

[2] L. Durlofsky, J. F. Brady, and G. Bossis, "Dynamic simulation of hydrodynamically interacting particles," Journal of Fluid Mechanics, vol. 180, pp. 21-49, 1987.

[3] M. Beiser, "Sedimentation submikroner Partikel in Abhängigkeit physikalisch-chemischer Einfflüsse und ihr Separationsverhalten in Dekantierzentrifugen," Dissertation, Universität Karlsruhe (TH), Germany, 2006.

[4] M. Beiser, G. Bickert, and P. Scharfer, "Comparison of sedimentation behavior and structure analysis with regard to destabilization processes in suspensions," Chemical Engineering \& Technology, vol. 27, no. 10, pp. 1084-1088, 2004.

[5] G. Bickert, "Sedimentation feinster suspendierter Partikeln im Zentrifugalfeld,” Dissertation, Universität Karlsruhe (TH), Germany, 1997.

[6] J. Happel and H. Brenner, Low Reynolds Number Hydrodynamics, Martinus Nijhoff Publishers, The Hague, The Netherlands, 3rd edition, 1983.
[7] G. K. Batchelor, "The stress system in a suspension of forcefree particles," Journal of Fluid Mechanics, vol. 41, no. 3, pp. 545-570, 1970.

[8] P. Kumar and B. V. Ramarao, "Enhancemant of the sedimentation rates of fribrous suspensions," Chemical Engineering Communications, vol. 108, no. 1, pp. 381-401, 1991.

[9] J. E. Butler and E. S. G. Shaqfeh, "Dynamic simulations of the inhomogeneous sedimentation of rigid fibres," Journal of Fluid Mechanics, vol. 468, pp. 205-237, 2002.

[10] B. Herzhaft and É. Guazzelli, "Experimental study of the sedimentation of dilute and semi-dilute suspensions of fibres," Journal of Fluid Mechanics, vol. 384, pp. 133-158, 1999.

[11] O. G. Harlen, R. R. Sundararajakumar, and D. L. Koch, "Numerical simulations of a sphere settling through a suspension of neutrally buoyant fibres," Journal of Fluid Mechanics, vol. 388, pp. 355-388, 1999.

[12] M. Stolarski, C. Eichholz, B. Fuchs, and H. Nirschl, "Sedimentation acceleration of remanent iron oxide by magnetic flocculation," China Particuology, vol. 5, no. 1-2, pp. 145-150, 2007.

\section{AUTHOR CONTACT INFORMATION}

Markus Feist: Institut für Mechanische Verfahrenstechnik und Mechanik (MVM), Universität Karlsruhe (TH), Karlsruhe, Germany; markus.feist@mvm.uni-karlsruhe.de

Hermann Nirschl: Institut für Mechanische Verfahrenstechnik und Mechanik (MVM), Universität Karlsruhe (TH), Karlsruhe, Germany; hermann.nirschl@mvm.uni-karlsruhe.de

Jörg Wagner: Fachgebiet Papierfabrikation und Mechanische Verfahrenstechnik (PMV), Technische Universität Darmstadt, Darmstadt, Germany; jwagner@tmo.de

Georg Hirsch: Fachgebiet Papierfabrikation und Mechanische Verfahrenstechnik (PMV), Technische Universität Darmstadt, Darmstadt, Germany; hirsch@papier.tu-darmstadt.de

Samuel Schabel: Fachgebiet Papierfabrikation und Mechanische Verfahrenstechnik (PMV), Technische Universität Darmstadt, Darmstadt, Germany; schabel@papier.tu-darmstadt.de 